\title{
Camera Parameters Auto-Adjusting Technique for Robust Robot Vision
}

\author{
Huimin Lu, Student Member, IEEE, Hui Zhang, Shaowu Yang and Zhiqiang Zheng, Member, IEEE
}

\begin{abstract}
How to make vision system work robustly under dynamic light conditions is still a challenging research focus in computer/robot vision community. In this paper, a novel camera parameters auto-adjusting technique based on image entropy is proposed. Firstly image entropy is defined and its relationship with camera parameters is verified by experiments. Then how to optimize the camera parameters based on image entropy is proposed to make robot vision adaptive to the different light conditions. The algorithm is tested by using the omnidirectional vision in indoor RoboCup Middle Size League environment and the perspective camera in outdoor ordinary environment, and the results show that the method is effective and color constancy to some extent can be achieved.
\end{abstract}

\section{INTRODUCTION}

How to make vision system work robustly under dynamic light conditions is still a challenging research focus in computer/robot vision community [1]. There are mainly three approaches to achieve this goal, and they correspond to different layers of robot vision. The first one is in image processing layer, and it is to process and transform the images to achieve some kind of constancy, such as color constancy [2] by Retinex algorithm [3][4]. The second one is in image analyzing layer, and it is to analyze and understand the images robustly, such as designing adaptive or robust object recognition algorithms [5][6]. These two approaches have attract lots of researchers' interest, and lots of progresses have been achieved. The third one is in image acquiring layer and is always ignored by researchers, which is to output the images to describe the real scene as consistently as possible in different light conditions by auto-adjusting the camera parameters [7][8][9](in this paper, camera parameters are the image acquisition parameters, not the intrinsic or extrinsic parameters in camera calibration).

In this paper, we try to use the third approach to achieve the robustness and adaptability of camera's output under different light conditions for robust robot vision. We also want to provide an objective method for vision/camera setup by this research, for the cameras are usually set manually according to user's subjective experiences when coming into a totally new working environment. We define the image entropy as the optimizing goal of camera parameters adjustment, and propose a novel camera parameters autoadjusting technique based on image entropy. We will test our algorithm by using our omnidirectional vision system [10] in the indoor RoboCup Middle Size League(MSL) environment

The authors are with Department of Automatic Control, College of Mechtronics and Automation, National University of Defense Technology, Changsha, Hunan, China. (phone: 86-731-84576455; email: $\{1$ hmnew, huizhang_nudt, ysw_nudt, zqzheng\}@ nudt.ed u. Cn) and the perspective camera in outdoor ordinary environment respectively.

In the following part, the related research will be introduced briefly in section II. We will present the definition of image entropy and verify that the image entropy is valid to represent the image quality for image processing and to indicate that whether the camera parameters are well set by experiments in section III, and then propose how to auto-adjust the camera parameters based on image entropy to adapt to the different illumination in section IV. The experimental results in indoor and outdoor environment and the discussions will be presented in section $\mathrm{V}$ and section VI respectively. The conclusion will be given in section VII finally.

\section{RELATED RESEARCH}

In the digital still cameras and consumer video cameras, many parameters adjusting mechanisms have been developed to achieve good imaging results, such as auto exposure by changing the iris or the shutter time [11], auto white balance [12], and auto focus [13]. In some special multiple slope response cameras, the response curve can be adjusted to adapt the dynamic response range to different light conditions by automatic exposure control [14]. But these methods are always on the camera hardware level, so we can not do these things or make modification on most cameras used in robot vision system except some special hardware-support cameras.

Some other related research took place in RoboCup especially MSL society which is a standard real-world test bed for robot vision and other relative research subjects. The final goal of RoboCup is that robot soccer team defeats human champion, so robots will have to be able to play competition in dynamic light conditions even in outdoor environment. So designing robust vision system is critical for robot's performance and RoboCup's final goal. Besides adaptive color segmentation methods [5], color online learning algorithms [15][16], and object recognition methods independent on color information [17][18], several researchers also have tried to adjust camera parameters to help achieving the robustness for vision sensors. Paper [7] defined the camera parameters adjustment as an optimization problem, and used the genetic meta-heuristic algorithm to solve it by minimizing the distance between the color values of some image areas and the theoretic values in color space. The theoretic color values were used as referenced values, so the effect from illumination could be eliminated, but the special image areas needed to be selected manually by users in the method. Paper [8] used a set of PID controllers to modify the intrinsic 
camera parameters like gain, iris, and two white balance channels according to the changes of a white reference color always visible in the omnidirectional vision system. Paper [9] adjusted the shutter time by designing a PI controller to modify the reference green field color to be the desired color values. Some reference color is needed in these three methods, so they are limited to be applied in other more situations.

\section{IMAGE ENTROPY AND ITS RELATIONSHIP WITH CAMERA PARAMETERS}

The setting of camera parameters affects the quality of outputting images greatly. Taking the cameras of our omnidirectional vision system as the example, only exposure time and gain can be adjusted (auto white balance has been realized in the camera, so we don't consider white balance). If the parameters are not properly set, the images could be less-exposed or over-exposed. These images can't represent the environments well, and we can say that the information content in these images is less than that in the well-exposed images. So both less-exposure and over-exposure will cause the loss of image information [19].

According to Shannon's information theory, the information content can be measured by entropy, and entropy increases with the information content. So we use image entropy to measure the image quality, and we also assume that the entropy of outputting images can indicate that whether the camera parameters are properly set. In the following part of this section, we will firstly present the definition of image entropy, and then verify this assumption by analyzing the distribution of image entropy with different camera parameters.

\section{A. The Definition of Image Entropy}

We use Shannon's entropy to definite the image entropy. Because RGB color space is a linear color space that formally uses single wavelength primaries and the color values are obtained directly after the CCD sensing of color cameras, it is more appropriate to calculate image entropy in RGB color space than in YUV or HSV color space. So the image entropy can be expressed as follows:

$$
\begin{aligned}
\text { Entropy }= & -\sum_{i=0}^{L-1} P_{R i} \log P_{R i}-\sum_{i=0}^{L-1} P_{G i} \log P_{G i} \\
& -\sum_{i=0}^{L-1} P_{B i} \log P_{B i}
\end{aligned}
$$

Where $L=256$ is the discrete level of RGB color channels, and $P_{R i}, P_{G i}, P_{B i}$ are the probability of color $R i, G i, B i$ existing in the image, and they can be replaced with frequency approximately and then calculated according to the histogram distribution of RGB color channels.

According to the definition in equation (1), $0=$ $\operatorname{Min}($ Entropy $) \leq$ Entropy $\leq \operatorname{Max}($ Entropy $)=-3 *$ $\sum_{i=0}^{255}(1 / 256) \log (1 / 256)=16.6355$, and the entropy will increase monotonously with the degree of average distribution of color values.

\section{B. Image Entropy's Relationship with Camera Parameters}

We capture a series of images by using our omnidirectional vision system in indoor environment and a perspective camera in outdoor environment with different exposure time and gain, and then calculate image entropy according to equation (1) to see how image entropy varies with camera parameters. The indoor environment is a standard RoboCup MSL field with dimension of $18 \mathrm{~m} * 12 \mathrm{~m}$, but the illumination is not only determined by the artificial lights, but also can be influenced greatly by natural light through lots of windows. The outdoor environment includes one blue patch, one black patch, and two orange balls near a small garden. All the experiments of this paper are performed in these two environments.

In the experiment of indoor environment, the range of exposure time is from $5 \mathrm{~ms}$ to $40 \mathrm{~ms}$ and the range of gain is from 5 to 22 . The experiment time of this section is evening, and the illumination is not affected by natural light. In the experiment of outdoor environment, the range of exposure time is from $1 \mathrm{~ms}$ to $22 \mathrm{~ms}$ and the range of gain is from 1 to 22 . The weather is cloudy, and the experiment time is midday. The minimal adjusting step of these two parameters is $1 \mathrm{~ms}$ and 1 respectively. We captured one image with each group of parameters. The image entropies changing with different camera parameters in the two experiments are shown in Figure 1 and Figure 2.

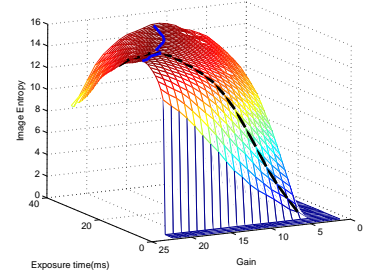

(a)

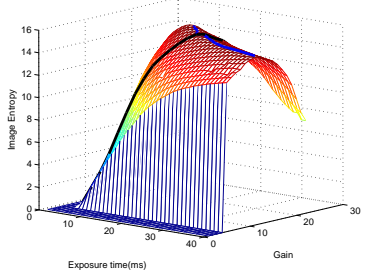

(b)
Fig. 1: The image entropies with different exposure time and gain in indoor environment. (a) and (b) are the same result viewed from two different view angles.

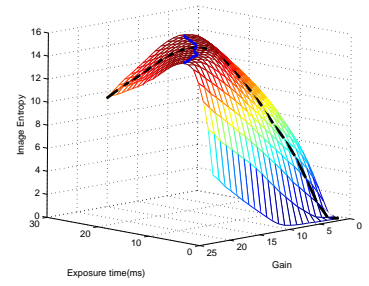

(a)

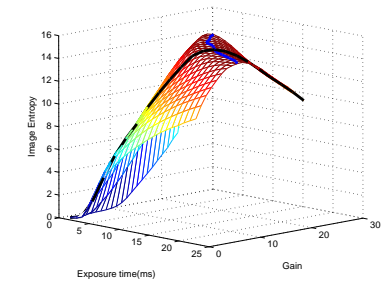

(b)
Fig. 2: The image entropies with different exposure time and gain in outdoor environment. (a) and (b) are the same result viewed from two different view angles.

From Figure 1 and 2, we can find that the manner in which image entropy varies with camera parameters is the same in the two experiments, and there is ridge curve (the blue curve in Figure 1 and 2). Along the ridge curve, the image entropies are almost the same in each experiment, and there 
is not obvious maximal value. So which image entropy along the ridge curve indicates the best image, or whether all the images related to the image entropy along the ridge curve are good?

For the images are used to processed and analyzed to realize object recognition, self-localization or other robot vision task, we test the quality of images by using the same color calibration result learned from one image [20] corresponding to a certain entropy on the ridge curve to segment the images corresponding to all the entropies along the ridge curve. In the indoor environment, we also detect the white line points using the algorithm proposed in paper [6], and they are very important for soccer robot's visual selflocalization. The typical images along the ridge curve and the processing results in the two experiments are demonstrated in Figure 3 and Figure 4.

As shown in the two figures, the images can be well segmented by the same color calibration result in each experiment, and object recognition can be realized successfully for robots. The same processing results are achieved in all the other images related to the image entropy along the ridge curve. So all these images are good for robot vision, and there is some kind of color constancy in these images, though they are captured under different camera parameters. It also means that all the setting of exposure times and gains corresponding to the image entropy along the ridge curve are acceptable for robot vision. So the assumption is verified that the image entropy can indicate that whether the camera parameters are properly set.
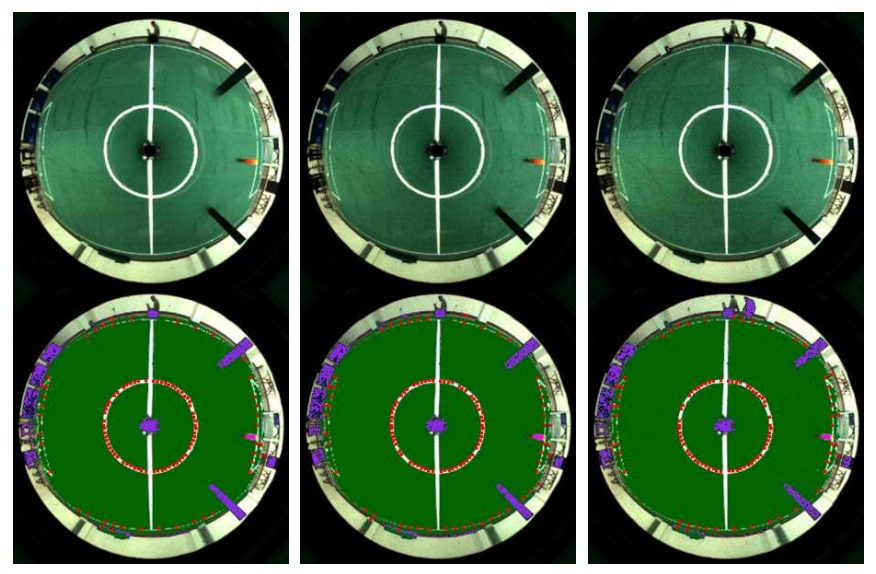

Fig. 3: The typical images along the ridge curve and the processing results in indoor experiment. (top) are the typical images. (bottom) are the processing results, and the red points are the detected white line points. The camera parameters are as follows: (left) exposure time: $34 \mathrm{~ms}$, gain: 13 . (middle) exposure time: $18 \mathrm{~ms}$, gain: 18 . (right) exposure time: 14ms, gain: 21.

\section{AUTO-ADJUSTING CAMERA PARAMETERS BASED ON IMAGE ENTROPY}

According to the experiments and analysis in last section, image entropy can indicate the image quality for robot vision and that whether the camera parameters are properly set, so camera parameters adjustment can be defined as an
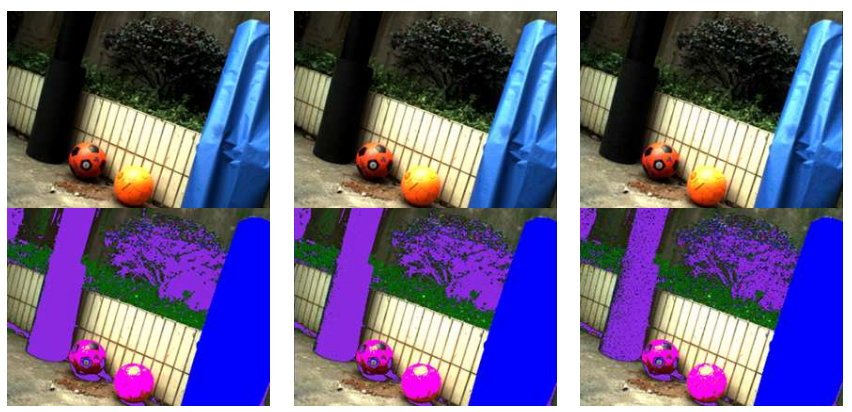

Fig. 4: The typical images along the ridge curve and the processing results in outdoor experiment. (top) are the typical images. (bottom) are the processing results. The camera parameters are as follows: (left) exposure time: $22 \mathrm{~ms}$, gain: 9 . (middle) exposure time: $14 \mathrm{~ms}$, gain: 14. (right) exposure time: $7 \mathrm{~ms}$, gain: 22 .

optimization problem, and image entropy can be used as optimizing goal. But as is shown in figure 1 and 2, the image entropies along the blue ridge curve are almost the same, and it is not easy to search the global optimal solution. Furthermore, camera parameters themselves will affect the performance of vision systems. For example, the real-time ability will decrease as exposure time increases, and the image noise will increase as gain increases. So exposure time and gain themselves have to be taken into account in this optimization problem. But it is difficult to measure the degree of these parameters' effect, so it is almost impossible to add some indicative or constraint function to image entropy directly for the optimization problem.

Considering that the images related to the image entropies along the ridge curve are all good for robot vision, we turn the two-dimension optimization problem to be onedimension one by defining some searching path. In this paper, we define the searching path as exposure time=gain (just equal in number value, for the unit of exposure time is $\mathrm{ms}$, and there is no unit for gain) to search the maximal image entropy in this path, and the camera parameters corresponding to the maximal image entropy are best for robot vision in current environment and current light condition. The searching path is shown as the black curve in figure 1 and 2 respectively in indoor and outdoor environment. The distributions of image entropy along the path in the two environments are demonstrated in Figure 5.

From Figure 5, a very good property of image entropy can be found that the image entropy will increase monotonously to the peak and then decrease monotonously along the defined searching path. So the global maximal image entropy can be found easily by searching along the defined path, and the best camera parameters are also determined at the same time. In Figure 5(a), the best exposure time and gain for the omnidirectional vision system are $18 \mathrm{~ms}$ and 18 respectively; in Figure 5(b), the best exposure time and gain for the perspective camera are $9 \mathrm{~ms}$ and 9 respectively.

In the real application, a reference image area should be determined, so robot can judge that whether it comes into a totally new environment or the illumination changes in the current environment by calculating the mean brightness value 


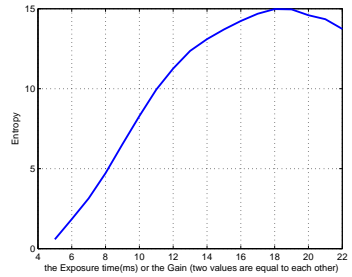

(a)

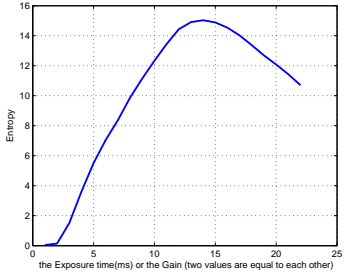

(b)
Fig. 5: The distribution of image entropy along the defined searching path. (a) The distribution in indoor environment. (b) The distribution in outdoor environment.

on the image area. For omnidirectional vision, according to its special character that the robot itself will be imaged in the central area of the panoramic images, this image area is used as reference area. For perspective camera, some special object should be recognized and tracked and then used as reference image area, such as the orange balls in figure 4 . If the increase of the mean value is higher than a threshold, the robot will consider that the illumination becomes stronger, and the optimization of camera parameters will be run towards the direction that exposure time and gain reduce and along the searching path. Similarly, if the decrease of the mean value is higher than the threshold, the optimization will be run towards the direction that exposure time and gain raise and along the searching path. In our experiment, we set the threshold as 20 . In the optimizing process, a new group of parameters will be set into the camera, and then a new image will be captured and the image entropy can be calculated according to equation (1). The new entropy will be compared with the last one to check whether the maximal entropy has reached. This iteration will go on and on until the maximal entropy is reached. About how to choose new parameters, the technique of varying optimizing step could be used to accelerate the optimization process. When the current entropy is not far from Max (Entropy), the optimizing step could be 1 , which means that the change of exposure time is $1 \mathrm{~ms}$ and the change of gain is 1 . When the current entropy is far from Max(Entropy), the optimizing step could be 2 or 3 .

The searching path can be changed according to different requirement about the vision system in different application. In some cases, the signal noise ratio of image is required to be high and the real-time performance is not necessary, so the searching path could be exposure time $=\alpha^{*}$ gain (also just equal in number value), and $\alpha>1$. In some other application, the camera is required to output image as soon as possible and the image noise is not restricted too much, so the searching path could be exposure time $=\alpha^{*}$ gain (also equal in number value), and $\alpha<1$.

\section{THE EXPERIMENTAL RESULTS}

In this section, we test our novel camera parameters autoadjusting algorithm proposed in last section under different light conditions in indoor environment and outdoor environment respectively. We verify that whether the camera parameters are properly set successfully by processing the images using the same color calibration result learned in the experiments of section III.

\section{A. The Experiments in Indoor Environment}

Two experiments are carried out in the indoor environment. In the first experiment, the weather is cloudy, and the experiment time is midday, so the illumination is influenced by artificial and natural light. The outputting image and the processing result are shown in Figure 6 when camera is set with the best parameters in section IV. The image is overexposed, and processing result is terrible. After the parameters have been optimized by our method, the outputting image and the processing result are demonstrated in Figure 7(a) and (b). The distribution of image entropy along the searching path is shown in Figure 7(c). The optimal exposure time is $13 \mathrm{~ms}$ and gain is 13 , so the image is well-exposed, and the processing result is also good. When we change the illumination gradually by turning off some lamps, the similar results are achieved.

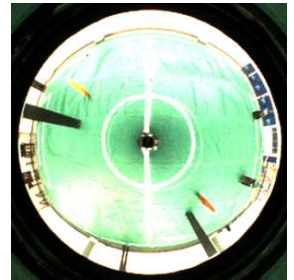

(a)

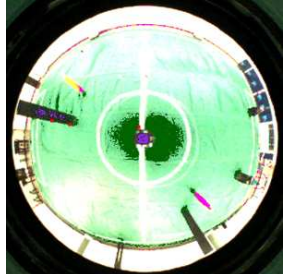

(b)
Fig. 6: (a) The outputting image when the camera parameters have not been optimized in indoor environment and the best parameters in section IV are used. (b) The processing result.

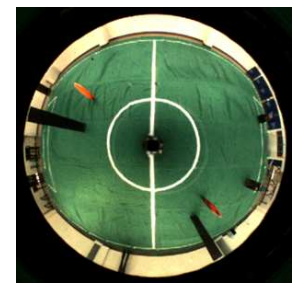

(a)

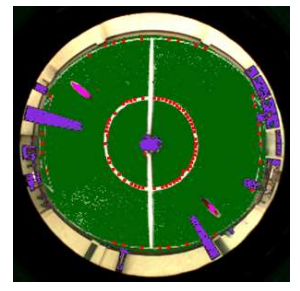

(b)

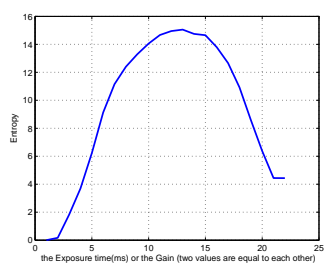

(c)

Fig. 7: (a) The outputting image after camera parameters have been optimized in indoor environment. (b) The processing result. (c) The distribution of image entropy along the searching path.

In the second experiment, we compare the robot's selflocalization results based on omnidirectional vision [21] with 
optimized camera parameters under very different illumination in three cases. In the first case, the light condition is the same as that in the experiment of section III. In the second case, the illumination is affected by strong sun's rays through the windows in a sunny day, and the optimal exposure time and gain are $12 \mathrm{~ms}$ and 12 respectively. In the third case, the weather and the experiment time are similar to those in the first experiment, but we change the illumination dynamically during robot's localization process by turning off and on the lamps, so the camera parameters will be auto-adjusted in real-time when robot detects that the illumination changes. The robot's self-localization results in these three cases are demonstrated in Figure 8. In the experiment, the robot is pushed by human to follow some straight traces on the field shown as black lines in Figure 8. The statistic of localization errors is shown in Table 1. The robot can achieve good localization results with the same color calibration result even under very different and dynamic light conditions. If the camera parameters are not adjusted according to the changes of illumination, robot's self-localization fails using the same color calibration result in the latter two cases. This experiment also verifies that our camera parameters adjusting method is effective for robot vision.

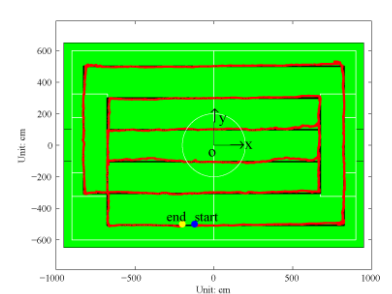

(a)

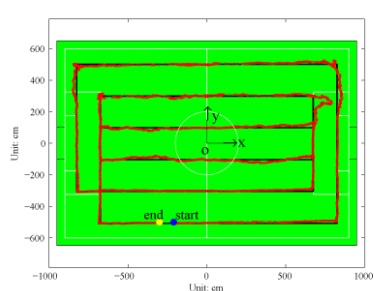

(b)

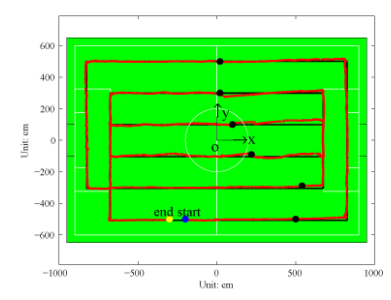

(c)

Fig. 8: The comparison of robot's localization with the same color calibration result after the camera parameters have been optimized under totally different illumination. (a) The result when the illumination is not affected by natural light. (b) The result when the illumination is affected greatly by the strong sun's rays. (c) The result when the illumination changes dynamically. The black points are the positions where the illumination changes and the camera parameters are auto-adjusted.

\section{B. The Experiment in Outdoor Environment}

In this experiment, the weather is sunny, and the experiment time is from midday to dusk, so the illumination is from bright to dark decided by natural light. We also use the same color calibration result in the outdoor experiment of section III to process the images. The outputting image and
TABLE I: The statistic of robot's self-localization errors under different illumination. In this table, $x, y$, are $\theta$ the self-localization coordinate related to the location $\mathrm{x}, \mathrm{y}$ and orientation.

\begin{tabular}{|c|c|c|c|c|}
\hline & & $x(\mathrm{~cm})$ & $y(\mathrm{~cm})$ & $\theta(\mathrm{rad})$ \\
\hline \multirow{3}{*}{ the first case } & mean error & 5.907 & 5.967 & 0.044 \\
\cline { 2 - 5 } & standard dev & 7.334 & 7.117 & 0.052 \\
\cline { 2 - 5 } & maximal error & 30.724 & 35.595 & 0.286 \\
\hline \multirow{3}{*}{ the second case } & mean error & 6.416 & 5.544 & 0.067 \\
\cline { 2 - 5 } & standard dev & 12.431 & 7.381 & 0.093 \\
\cline { 2 - 5 } the thrid case & maximal error & 95.396 & 33.063 & 0.580 \\
\hline & mean error & 2.751 & 5.867 & 0.047 \\
\cline { 2 - 5 } & standard dev & 3.593 & 7.533 & 0.061 \\
\cline { 2 - 5 } & maximal error & 16.834 & 35.173 & 0.279 \\
\hline
\end{tabular}

the processing result are shown in Figure 9 when camera is set with the best parameters in section IV. The image is over-exposed, and processing result is unacceptable for robot vision. After the parameters have been optimized, the outputting image and the processing result are demonstrated in Figure 10(a) and (b). The distribution of image entropy along the searching path is shown in Figure 10(c). The optimal exposure time is $9 \mathrm{~ms}$ and gain is 9 , so the image is well-exposed, and the processing result is also good. We also process the images captured with some suboptimal camera parameters, and the results are demonstrated in Figure 11. All the color classification results in Figure 11 are more or less worse than that in Figure 10, so it also verifies that the image captured with the optimal camera parameters is the optimal image for robot vision. When the experiment is run in different time from midday to dusk, all images can be well-exposed and well processed after the camera parameters have been optimized.

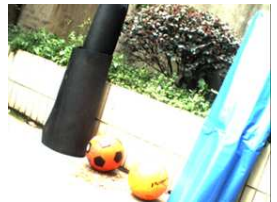

(a)

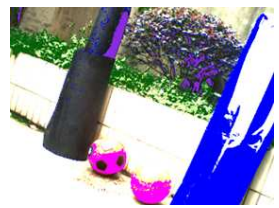

(b)
Fig. 9: (a) The outputting image when the camera parameters have not been optimized in outdoor environment and the best parameters in section IV are used. (b) The processing result.

\section{DISCUSSION}

According to the analysis and the experimental results in the above sections, our camera parameters auto-adjusting method based on image entropy can make the camera's output adaptive to different light conditions and describe the real world as consistently as possible. So the color constancy to some extent for the vision system is achieved. Furthermore, unlike other existing methods mentioned in section II, there is not any reference color needed during the optimization process of our method, so our method can be applied in much more situations. Our method also provides an objective vision/camera setup technique when robots come into a totally new working environment, so users don't need to adjust camera parameters manually according to experience. 


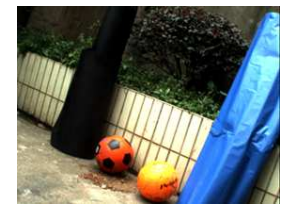

(a)

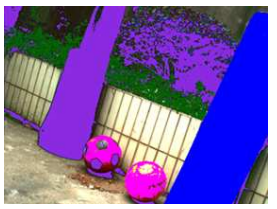

(b)

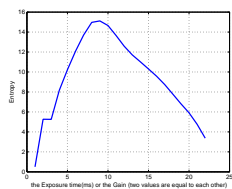

(c)

Fig. 10: (a) The outputting image after camera parameters have been optimized in outdoor environment. (b) The processing result. (c) The distribution of image entropy along the searching path.

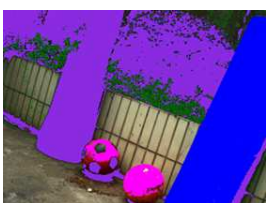

(a)

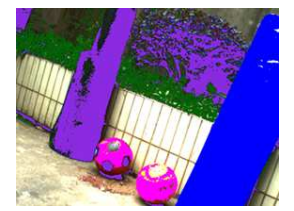

(c)

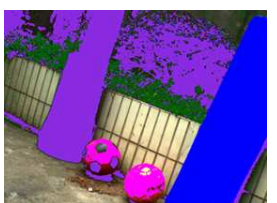

(b)

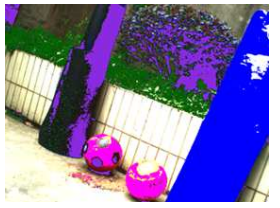

(d)
Fig. 11: The processing results of images captured with some suboptimal camera parameters in outdoor environment. (a) exposure time: 7ms, gain: 7. (b) exposure time: $8 \mathrm{~ms}$, gain: 8 . (c) exposure time: $10 \mathrm{~ms}$, gain: 10 . (d) exposure time: $11 \mathrm{~ms}$, gain: 11 .

Besides exposure time and gain adjusted in above experiments, our method can be extended to adjust more parameters if supported by hardware. We replace the original lens of our perspective camera with HZC08080 lens, so the iris can be adjusted by sending commands to control the motors of lens in software. The distribution of image entropy with different iris and exposure time, the image entropies along the defined searching path and the optimal image along this path are shown in Figure 12.

About the real-time performance of our method, for the light condition will not change too suddenly in real application, it only takes several cycles to finish the optimizing process. And it takes about $40 \mathrm{~ms}$ to set the parameters into our camera for one time. So camera parameters adjustment can be finished in maximal several hundred $\mathrm{ms}$, and there is not problem for our method in real-time requirement.

However, there are still some deficiencies in our algorithm. For example, our method can not deal with the situation that the illumination is highly not uniform. Because image entropy is a global appearance feature for image, it may be not the best optimizing goal in this situation. As shown in Figure 13, though the camera parameters have been

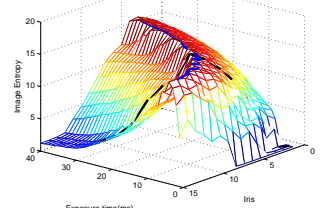

(a)

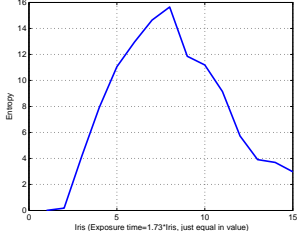

(b)

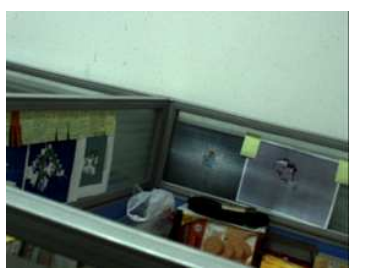

(c)

Fig. 12: (a) The distribution of image entropy with different iris and exposure time. (b) The image entropies along the defined searching path. (c) The optimal image along the searching path.

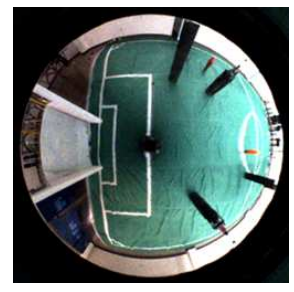

(a)

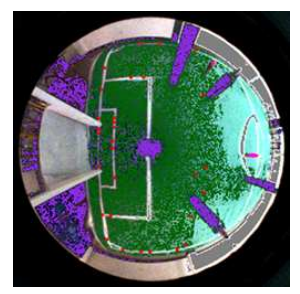

(b)

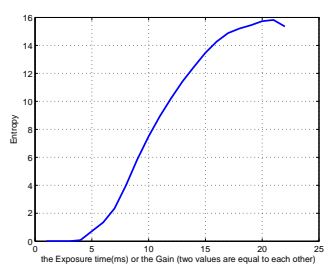

(c)

Fig. 13: (a) The outputting image after camera parameters have been optimized when the illumination is highly not uniform and robot is located in very dark place. (b) The processing result. (c) The distribution of image entropy along the searching path.

optimized as $21 \mathrm{~ms}$ and 21 , but the image processing result is still unacceptable for robot vision. Object recognition or tracking technique should be integrated in our method, and camera parameters can be optimized according to local image entropy or other features near the object area on the images.

\section{CONCLUSION}

In this paper, a novel camera parameters auto-adjusting method is proposed to make camera's output adaptive to different light conditions for robust robot vision. Firstly we present the definition of image entropy, and use image entropy as optimizing goal for the optimization problem of camera parameters after verifying that image entropy can indicate whether the camera parameters are properly set by experiments. Then how to optimize the camera parameters 
for robot vision based on image entropy is proposed to adapt to different illumination. The experiments in indoor RoboCup MSL standard field and outdoor ordinary environment show that our algorithm is effective and the color constancy to some extent in the output of vision systems can be achieved.

\section{REFERENCES}

[1] G. Mayer, H. Utz, and G.K. Kraetzschmar, "Playing Robot Soccer under Natural Light: A Case Study", RoboCup 2003: Robot Soccer World Cup VII, pp. 238-249, 2004.

[2] V. Agarwal, B.R. Abidi, A. Koschan, and M.A. Abidi, "An Overview of Color Constancy Algorithms", Journal of Pattern Recognition Research, vol.1, no.1, pp. 42-54, 2006.

[3] D.A. Forsyth, "A Novel Algorithm for Color Constancy", International Journal of Computer Vision, vol.5, no.1, pp. 5-35, 1990.

[4] G. Mayer, H. Utz, and G.K. Kraetzschmar, "Towards Autonomous Vision Self-calibration for Soccer Robots", in Proc. of the IEEE/RSJ International Conference on Intelligent Robots and Systems (IROSO2), pp. 214-219, 2002.

[5] C. Gönner, M. Rous, and K. Kraiss, "Real-Time Adaptive Colour Segmentation for the RoboCup Middle Size League", RoboCup 2004: Robot Soccer World Cup VIII, pp. 402-409, 2005.

[6] H. Lu, Z. Zheng, F. Liu, and X. Wang, "A Robust Object Recognition Method for Soccer Robots", in Proc. of the 7th World Congress on Intelligent Control and Automation, pp. 1645-1650, 2008.

[7] E. Grillo, M. Matteucci, and D.G. Sorrenti, "Getting the most from your color camera in a color-coded world", RoboCup 2004: Robot Soccer World Cup VIII, pp. 221-235, 2005.

[8] Y. Takahashi, W. Nowak, and T. Wisspeintner, "Adaptive Recognition of Color-Coded Objects in Indoor and Outdoor Environments", RoboCup 2007: Robot Soccer World Cup XI, pp. 65-76, 2008.

[9] J.J.M. Lunenburg, and G.V.D. Ven, "Tech United Team Description", in RoboCup 2008 Suzhou, CD-ROM, 2008.

[10] H. Lu, H. Zhang, J. Xiao, F. Liu, and Z. Zheng, "Arbitrary Ball Recognition Based on Omni-directional Vision for Soccer Robots", RoboCup 2008: Robot Soccer World Cup XII, pp. 133-144, 2009.

[11] T. Kuno, H. Sugiura, and N. Matoba, "A New Automatic Exposure System for Digital Still Cameras", IEEE Transactions on Consumer Electronics, vol.44, no.1, pp. 192-199, 1998.

[12] V. Chikane, and C. Fuh, "Automatic White Balance for Digital Still Cameras", Journal of Information Science and Engineering, vol.22, no.3, pp. 497-509, 2006.

[13] N. Ng Kuang Chern, P.A. Neow, and M.H. Ang Jr, "Practical Issues in Pixel-Based Autofocusing for Machine Vision", in Proc. of the 2001 IEEE International Conference on Robotics and Automation, pp. 27912796, 2001.

[14] A. Goo $\beta$ en, M. Rosenstiel, S. Schulz, and R. Grigat, "Auto Exposure Control for Multi-Slope Cameras", in Proc. of ICIAR 2008, pp. 305314,2008

[15] F. Anzani, D. Bosisio, M. Matteucci, and D.G. Sorrenti, "On-Line Color Calibration in Non-Stationary Environments", RoboCup 2005: Robot Soccer World Cup IX, pp. 396-407, 2006.

[16] P. Heinemann, F. Sehnke, F. Streichert, and A. Zell, "Towards a Calibration-Free Robot: The ACT Algorithm for Automatic Online Color Training", RoboCup 2006: Robot Soccer World Cup X, pp. 363370, 2007.

[17] R. Hanek, T. Schmitt, S. Buck, M. Beetz, "Towards RoboCup without Color Labeling", RoboCup 2002: Robot Soccer World Cup VI, pp. 179194, 2003.

[18] A. Treptow, and A. Zell, "Real-time object tracking for soccerrobots without color information", Robotics and Autonomous Systems, vol.48, no. 1, pp. 41-48, 2004.

[19] A.A. Goshtasby, "Fusion of Multi-exposure images", Image and Vision Computing, vol.23, no.6, pp. 611-618, 2005.

[20] F. Liu, H. Lu, and Z. Zheng, "A Modified Color Look-Up Table Segmentation Method for Robot Soccer", in Proc. of the 4th IEEE LARS/COMRob 07, 2007.

[21] H. Zhang, H. Lu, X. Wang, et al., "NuBot Team Description Paper 2008", in RoboCup 2008 Suzhou, CD-ROM, 2008. 